\title{
The rising crisis of illicit fentanyl use, overdose, and potential therapeutic strategies
}

\author{
Ying Han', Wei Yan², Yongbo Zheng², Muhammad Zahid Khan', Kai Yuan² and Lin Lư
}

\begin{abstract}
Fentanyl is a powerful opioid anesthetic and analgesic, the use of which has caused an increasing public health threat in the United States and elsewhere. Fentanyl was initially approved and used for the treatment of moderate to severe pain, especially cancer pain. However, recent years have seen a growing concern that fentanyl and its analogs are widely synthesized in laboratories and adulterated with illicit supplies of heroin, cocaine, methamphetamine, and counterfeit pills, contributing to the exponential growth in the number of drug-related overdose deaths. This review summarizes the recent epidemic and evolution of illicit fentanyl use, its pharmacological mechanisms and side effects, and the potential clinical management and prevention of fentanyl-related overdoses. Because social, economic, and health problems that are related to the use of fentanyl and its analogs are growing, there is an urgent need to implement large-scale safe and effective harm reduction strategies to prevent fentanyl-related overdoses.
\end{abstract}

\section{Introduction}

Fentanyl was first developed in 1960 by Paul Janssen as a potent opioid anesthetic and analgesic. At the time, fentanyl was the fastest-acting opioid discovered to date and more powerful than morphine (50-100 times) and heroin $(30-50 \text { times })^{1,2}$. Transdermal, intravenous, and transbuccal fentanyl administration and several other drugs with chemical structures that are similar to fentanyl have been developed, approved, and used for surgical anesthesia and the management of severe cancer pain and perioperative pain, eventually becoming the most often used synthetic opioid in clinical practice ${ }^{3-5}$. Since 1979, fentanyl and its analogs have been synthesized in laboratories and sold as heroin substitutes or mixed with other illicitly sourced drugs, leading to an increase in fentanyl-related overdose deaths ${ }^{6,7}$. Postmortem studies have consistently found pulmonary edema, congestion,

\footnotetext{
Correspondence: Lin Lu (linlu@bjmu.edu.cn)

${ }^{1}$ National Institute on Drug Dependence and Beijing Key Laboratory of Drug Dependence, Peking University, 100191 Beijing, China

${ }^{2}$ Peking University Sixth Hospital, Peking University Institute of Mental Health, NHC Key Laboratory of Mental Health (Peking University), National Clinical Research Center for Mental Disorders (Peking University Sixth Hospital), 100191 Beijing, China

Full list of author information is available at the end of the article.
}

and needle puncture sites in these victims. Based on data from the National Vital Statistics System, 599,255 drug overdose deaths occurred from 1979 to 2016 in the United States, and the overall mortality rate has seen exponential growth. Fentanyl-related overdose deaths predominantly occurred in the northeastern United States, mostly affecting younger people (20-40 years of age), and grew sharply since $2013^{8}$.

Rapid death from ingesting fentanyl has become increasingly more common. Its high potency, fast onset of action, and duration of the desired effect may be particularly important contributing factors to the higher risk of overdose deaths and social consequences ${ }^{9}$. Fentanyl has become a major contributor to cocaine-related fatal overdoses. The rate of fentanyl-related overdose deaths increased 55\% between 2015 and 2017 in New York city $^{10,11}$. Synthetic opioids are also increasingly detected in illicit supplies of heroin, methamphetamine, and counterfeit pills. Analysis of a sampling of 1 million unique patients' urine drug test (UDT) specimens showed that positivity rates for fentanyl have increased by $1850 \%$ among cocaine positive UDT results and increased by 798\% among methamphetamine-positive UDT results between January 2013 and September 2018 ${ }^{12}$. This 
mixture may lead to the increases in cocaine-related and methamphetamine-related overdoses. Moreover, the number of fatal overdoses from synthetic opioids, primarily fentanyl and its analogs, was 19,547 in 2016 in the United States, and this rate increased by $88 \%$ per year from 2013 to $2016^{13-16}$. The incidence of heroin-related overdose deaths stabilized in 2017, whereas deaths that involved other synthetic opioids continued to increase ${ }^{17}$. Given the substantial individual and public health threats of this emerging problem, the present review summarizes the epidemic and evolution of illicit fentanyl use, its pharmacological mechanism of action, its adverse consequences, and the clinical management and prevention of fentanyl-related overdoses.

\section{Epidemic and evolution of illicit fentanyl use}

Fentanyl is currently approved and commonly used to treat breakthrough pain in cancer patients and various other clinical conditions that involve noncancer pain, such as postoperative pain. However, its potential for abuse and the rise in overdose deaths pose a serious challenge to public health ${ }^{18-21}$. Deaths that were attributable to illicit fentanyl use were first reported in the early 1980s and occurred sporadically in the United States ${ }^{6,7,22}$. A surge in the occurrence of fentanyl-related fatalities among illicit drug users occurred in 2006. A total of 1013 deaths in six states occurred from April 4, 2005, to March 28, $2007^{23}$. Since then, the prevalence of opioidrelated mortality has increased persistently, and the number of reported fentanyl-related deaths more than doubled (from 2628 to 5544) between 2012 and $2014^{21,24,25}$. The rate of fentanyl-related overdose deaths increased from $<15 \%$ in 2010 to $~ 50 \%$ in 2017 in Marion County, Indiana ${ }^{26}$. Overall overdose deaths and firstresponder calls increased in a community-based sample in an impoverished neighborhood in Vancouver, Canada, in 2017, and fentanyl was detected in $52 \%$ of the subjects who were prescribed opioid agonist therapy $y^{27}$. At the same time, fentanyl-related deaths also increased in Australia $^{28,29}$.

The presence of fentanyl and its analogs has become a central contributor to the increase in the number of opioid-related overdose deaths. Preliminary estimates of opioid overdose deaths in the United States in 2016 revealed that fentanyl and its analogs (e.g., acetylfentanyl, furanylfentanyl, and carfentanil) have contributed to nearly half of opioid overdose deaths ${ }^{16,30,31}$. Moreover, the number of deaths that were attributable to illicitly manufactured fentanyl and its analogs nearly quadrupled between July 2015 and June 2017 in Montgomery County, $\mathrm{Ohio}^{32}$. Heroin-positive cases declined while methamphetamine-positive cases increased in these victims. Urine drug screens showed that the prevalence of recent fentanyl use in patients who received opioid agonist treatment in England was 3\%, and multiple fatalities with synthetic fentanyl analogs were reported in northern England in early 2017 33,34 .

Fentanyl is $\sim 30-50$ times more potent than heroin, and smaller volumes of heroin and other drugs that are adulterated with fentanyl can produce powerful effects with lower production costs. Detecting fentanyl and its analogs in used syringes can reveal exposure risk ${ }^{35}$. The fentanyl detection rate was significantly higher among drug users who injected drugs in the past 6 months compared with non-injection drug users. The prevalence of non-fatal overdose is very high among people who inject drugs ${ }^{36-38}$. The prevalence of intravenous fentanyl use among people who inject drugs in Australia is $8 \%$. Given the narrow range between effective and lethal doses, this population is at high risk of overdose ${ }^{37,39,40}$. The opioid crisis is likely attributable to illicitly manufactured fentanyl and its analogs around the world, especially when they are mixed with heroin and other drugs, and the route of administration ${ }^{41,42}$.

Many people who have survived fentanyl overdose appear to be unaware that they ever took the drug. Surveys from 17 harm reduction sites in British Columbia, Canada, revealed that the prevalence of fentanyl use was $29 \%$ (70/242; based on urine drug screen), $73 \%$ of whom report that they did not knowingly use fentanyl ${ }^{43}$. Urine drug screens in methadone-maintained patients in Wayne County, Michigan, showed that $38 \%$ of 368 unique patients tested positive for fentanyl, and $67.3 \%$ of 113 patients reported that they did not know anyone who sought to obtain fentanyl in a subsequent anonymous survey $^{44}$. A high risk of overdose and deaths was found among this vulnerable population that exhibited high fentanyl exposure, thus highlighting the pressing need to develop appropriate harm-reduction strategies, such as surveillance, the development of early-warning systems, pill-testing technology about the presence of fentanyl in various drug products, naloxone training and distribution, overdose education, and urine screens ${ }^{21,45,46}$. The vast majority of people reported their willingness to use rapid test strips to detect the presence of fentanyl in drugs or urine at home or utilize drug-checking services at supervised injection clinics ${ }^{47,48}$. Multiplex ultrahighperformance liquid chromatography (UHPLC-MS)/ liquid chromatography tandem mass spectrometry (LC-MS-MS)/liquid chromatography-quadrupole timeof-flight-mass spectrometry (LC-QTOF-MS) analyses have also been developed and validated for the detection of fentanyl and its analogs and metabolites in blood, hair, and oral fluid, which will be helpful for informing harm reduction behaviors and combating the fentanyl crisis $^{49-52}$. A newly developed lateral flow immunoassay was also evaluated for effectiveness in the detection of fentanyl analogs ${ }^{53}$. 


\section{Pharmacological mechanisms and side effects of fentanyl}

Despite the beneficial clinical anesthetic and painrelieving effects of fentanyl, the frequent use of fentanyl primarily affects the central nervous system (CNS) and gastrointestinal, cardiovascular, and pulmonary systems and can cause several side effects ${ }^{54}$. Digestive symptoms, such as nausea, vomiting, and constipation, are common in patients who repeatedly use fentanyl ${ }^{55,56}$. Immunosuppression was also shown to be precipitated by analgesic opioid drugs, including fentanyl, in preclinical and clinical studies. Such immunosuppression can be especially dangerous in the elderly and already immunocompromised patients ${ }^{57-59}$. Additionally, fentanyl and synthetic opioids have other frequently reported side effects, including migraine, dizziness, vertigo, confusion, hallucinations, and a higher risk of fractures in the elderly ${ }^{59-63}$. Fentanyl has rewarding effects and thus high abuse potential. Its repeated use leads to the development of tolerance and drug dependence ${ }^{64,65}$. Analyses of adverse-event reporting systems in the United States, Europe, and the United Kingdom have shown that cases of fentanyl-related misuse, abuse, dependence, and withdrawal steadily increased between 2004 and 2018, resulting in prolonged hospitalization or death ${ }^{66}$. Other mental disorders, such as depression, insomnia, and suicidality, can also occur with fentanyl abuse, contributing to relapse and a higher risk of respiratory depression or overdose death ${ }^{65,67}$. The treatment of these mental disorders may help prevent fentanyl-related fatalities and achieve abstinence.

Fentanyl is a full $\mu$-opioid receptor agonist, but it also acts on $\delta$ - and $\kappa$-opioid receptors ${ }^{68,69}$. Fentanyl has been shown to exert its analgesic and lethal effects through different receptor populations in the CNS. It is eliminated from cerebrospinal fluid at approximately the same rate as morphine $\mathrm{e}^{70,71}$. Acute naloxone administration antagonizes fentanyl-induced analgesia more than fentanylinduced lethality. $\beta$-funaltrexamine was shown to inhibit both fentanyl-induced analgesia and lethality ${ }^{71}$. Overdoserelated concentrations of fentanyl were shown to block human ether-a-go-go-related gene (hERG) potassium channels in ventricular myocytes that were isolated from neonatal rats, which may contribute to fentanyl-related overdose death or sudden death ${ }^{72}$.

Respiratory depression is the most dangerous adverse reaction to fentanyl that can result in lethality. In rats, intravenous injections of fentanyl dose-dependently decreased oxygen levels in the nucleus accumbens, basolateral amygdala, and subcutaneous space, followed by a delayed increase in glucose and fluctuations in brain temperature and metabolic brain activity ${ }^{73-75}$. Neuronal hypermetabolism that is induced by fentanyl and its analogs may damage the hippocampus and limbic system, causing an amnestic syndrome in patients who use fen$\operatorname{tanyl}^{76-79}$. With regard to brain hypoxia and hypothermia, fentanyl has synergistic effects with heroin, which is consistent with the higher risk of overdose death that is associated with heroin-fentanyl mixtures ${ }^{73,80}$. Fentanylrelated respiratory depression is also dose-dependent, which reaches a peak 5 min after administration and requires $4 \mathrm{~h}$ to recover in humans. Such effects can lead to prolonged apnea and sudden death ${ }^{74,81,82}$. Epidural fentanyl infusion has been shown to cause postoperative adult respiratory distress syndrome ${ }^{83}$. The $\mu_{1}$-opioid receptor is involved in respiratory depression that is induced by fentanyl and its analogs but not morphine ${ }^{84}$. Selective $\alpha 4 \beta 2$ nicotinic receptor agonist A85380 reversed fentanyl-induced respiratory depression in rats without significant side effects ${ }^{85}$. The calcium-activated potassium channel blocker GAL021 was shown to attenuate morphine-induced respiratory depression in rats, mice, and nonhuman primates, and it produced stimulatory effects during alfentanil-induced respiratory depression, without affecting sedation in humans ${ }^{86-88}$. However, more studies are needed to confirm the efficacy and potential toxicity of A85380 and GAL021.

Many studies have reported cardiovascular symptoms after fentanyl-induced analgesia, such as myocardial ischemia, QTc interval prolongation, and bradycardia ${ }^{89-91}$. Fentanyl is commonly used during percutaneous coronary interventions, but the relative safety of its use requires further investigation because intravenous fentanyl has been reported to induce hypothermia, impair ticagrelor absorption, and cause antiplatelet effects ${ }^{92-94}$. Autopsy and toxicological analyses indicated that chronic fentanyl use may be responsible for hypertrophy, cardiac fibrosis, and atherosclerosis ${ }^{54,95,96}$. Neither sigma nor opioid receptors are essential for the fentanyl-induced attenuation of muscarinic coronary contraction ${ }^{97}$.

Fentanyl administration provides effective pain relief, but its long-term use can result in a lowering of pain thresholds ${ }^{98,99}$. This phenomenon of fentanyl-induced hyperalgesia is a challenge in the clinical management of perioperative and chronic pain. Recent studies showed that fentanyl-induced hyperalgesia was modulated by the activation of extracellular signal-regulated kinase in the laterocapsular division of the central nucleus of the amygdala (CeLC) and CaMKII $\alpha$ in the CeLC-periaqueductal gray-rostral ventromedial medulla-spinal cord descending facilitative pain pathway in rats $^{100,101}$.

\section{Interventions for the management and prevention of fentanyl overdose}

Similar treatments are prescribed for opioid use disorder and opioid overdose, including the Food and Drug Administration (FDA)-approved medications methadone, 
buprenorphine, extended-release naltrexone, and naloxone $^{102}$. Lofexidine, a central $\alpha_{2}$-adrenergic receptor agonist, was the first non-opioid medication that was approved by the United States FDA for the treatment of opioid withdrawal ${ }^{103,104}$. Lofexidine has fewer prescriptive barriers and comparable efficacy and safety relative to other opioid receptor agonizts, but it is generally more expensive. Sparse data are available on the effectiveness of interventions to prevent overdoses that are caused by illicitly manufactured fentanyl (Table 1). Compared with other opioid-related overdoses, illicit fentanyl-related overdoses appear to be accompanied by distinct symptoms, such as body and chest rigidity, dyskinesia, and slow or irregular heart rate, which can affect overdose management, such as oxygen provisions and appropriate doses of naloxone ${ }^{105,106}$. To avoid or reduce the adverse effects of fentanyl, the FDA proposed to control the duration of use and doses of fentanyl ${ }^{107}$. One study showed that the majority of patients who were presumed to experience fentanyl overdose could be discharged after brief emergency room observation, thus unlikely requiring additional naloxone dosing in the emergency room ${ }^{108}$.

There are limited data on the efficacy of methadone or buprenorphine for the treatment of illicit fentanyl use. A retrospective study in Rhode Island showed that 6 months of methadone maintenance protected against death and promoted abstinence in fentanyl-exposed patients, but relapse rates were still high ${ }^{109}$. Buprenorphine is a $\mu$ opioid receptor partial agonist and $\mathrm{K}$-opioid receptor antagonist that is commonly used to treat opioid use disorder. It also exerts antidepressant and anxiolytic activity and is a promising treatment for neonatal opioid withdrawal syndrome ${ }^{110}$. A retrospective cohort study showed that 6-month treatment retention rates and opioid abstinence rates were not different between individuals who were positive for fentanyl or heroin at baseline before initiating buprenorphine treatment, indicating that buprenorphine may still be beneficial for treating fentanyl exposure ${ }^{111}$. Repeated treatment with buprenorphine produced a greater magnitude of antinociceptive tolerance than higher-efficacy agonizts (e.g., morphine and etonitazene) in rats ${ }^{112}$. Studies in pigeons and rhesus monkeys showed that the amount of tolerance that develops to the reinforcing potency of opioids depends on their efficacy, and the higher-efficacy $\mu$-opioid receptor agonist sufentanil was more difficult to antagonize than the low-efficacy $\mu$-opioid receptor agonist morphine ${ }^{113-115}$. These data indicate that buprenorphine may have lower efficacy for the treatment of fentanyl overdose compared with heroin overdose, although no human trials have been performed to date ${ }^{116}$.

Naloxone is a $\mu$-opioid receptor antagonist that is used to treat fentanyl-related overdose, regardless of the suspected route of administration. However, its efficacy is

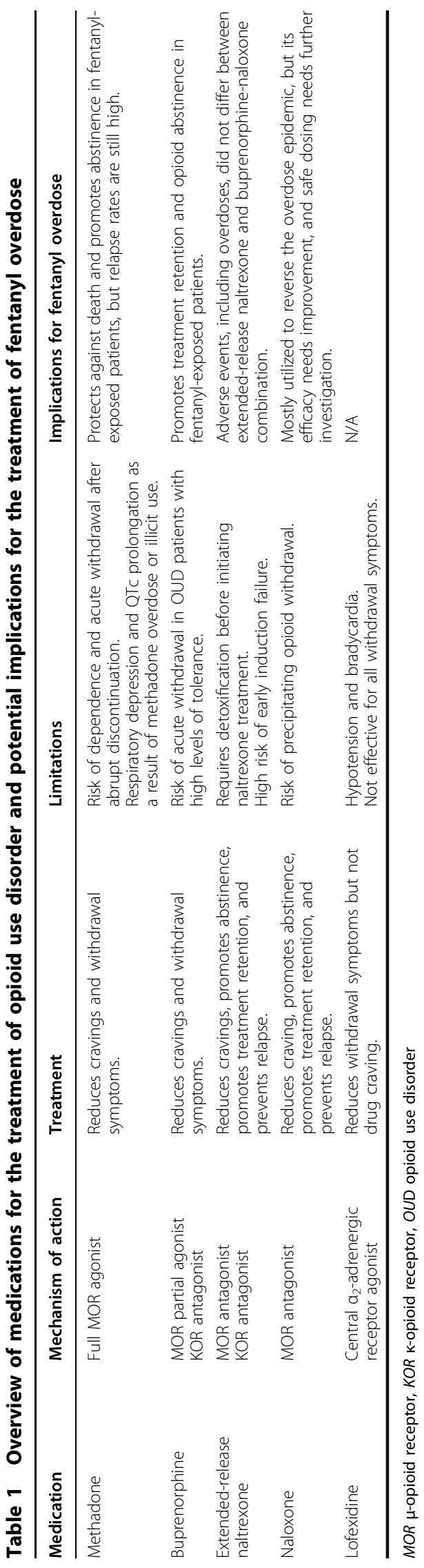


inconsistent, and safe dosing needs to be considered from the perspective of precipitating opioid withdrawal ${ }^{117-119}$. Recent studies also showed that extended-release naltrexone was equally safe and effective as a buprenorphine-naloxone combination at promoting abstinence and treatment retention once treatment was initiated, but fewer participants successfully initiated naltrexone treatment ${ }^{120,121}$. Larger or repeated doses of naloxone are speculated to be required for the treatment of fentanyl overdose because of its higher affinity for $\mu$ opioid receptors. However, a study of a community naloxone distribution program in Allegheny County showed that the average doses of naloxone that were administered to reverse overdose did not change between 2013 and 2016, although the incidence of overdoses that were related to fentanyl and its analogs increased during the same time ${ }^{122}$. A retrospective study of the fentanyl epidemic in Chicago showed that doses of naloxone up to $12 \mathrm{mg}$ may effectively treat fentanyl overdose ${ }^{123}$. Naloxone was shown to reverse transdermal fentanyl overdoseinduced sedation, the reduction of body temperature, and the reduction of heart rate in $\operatorname{dogs}^{124}$. A systematic review found a low incidence of mortality or serious adverse events that were caused by prehospital naloxone administration in opioid overdose patients, although the source of overdose was mostly heroin and not fentanyl ${ }^{125}$. Additionally, seeking emergency medical help was positively associated with overdose victims who received higher doses of naloxone and rescue breathing in British Columbia, Canada ${ }^{126}$. A survey of 316 street-recruited people who used opioids in Baltimore showed that the majority of them perceived the high risk of fentanyladulterated heroin and overdose, but most of them did not often carry naloxone with them ${ }^{127}$. The early adoption and distribution of take-home naloxone have been reported to effectively prevent opioid overdose deaths ${ }^{128-130}$. Therefore, harm reduction strategies, such as safe injection sites, the expansion of available opioid agonist treatment, and overdose prevention training (e.g., carrying naloxone and not use drugs alone, higher dose or multiple administrations of naloxone), are needed to control the adverse effects of fentanyl and reduce overdoses ${ }^{131}$.

Additionally, more potent, longer-acting opioid receptor antagonists are needed to prevent fentanyl-related overdose deaths. Compared with naloxone, nalmefene has been shown to have superior efficacy in reversing the carfentanil-induced loss of righting reflex and respiratory depression in rats $^{132}$. Nalmefene is generally well tolerated and is a recent option for patients with alcohol dependence ${ }^{133-135}$. Additionally, novel, selective, and potent $\mu$-opioid receptor antagonists, such as 17-cyclopropylmethyl-3,14 $\beta$-dihydroxy-4,5 $\alpha$-epoxy- $6 \alpha$ (isoquinoline-3-carboxamido)morphinan (NAQ) and 17-cyclopropylmethyl-3,14 $\beta$-dihydroxy-4,5 $\alpha$-epoxy- $6 \alpha$ - (indole-7-carboxamido)morphinan (NAN), have been reported to produce less opioid tolerance, dependence, and withdrawal signs. Furthermore, NAN pretreatment was shown to block the discriminative stimulus effects of fentanyl in rats. The orexin-1 receptor antagonist SB334867 was also shown to decrease motivation and demand for fentanyl in rats ${ }^{136}$. Therefore, these drugs could be considered candidates for the treatment of opioid use disorder ${ }^{137}$. Chronic anticonvulsant carbamazepine therapy was shown to increase fentanyl clearance and decrease plasma concentrations in neurosurgical patients, which may attenuate the actions of fentanyl ${ }^{138}$. A case report showed that treatment with slow-release oral morphine in a near-fatal fentanyl overdose patient was successful, despite the patient's previous failures with methadone and buprenorphine/naloxone-based opioid agonist therapies, which could be considered potential alternative treatments ${ }^{139}$.

Previous studies have reported the vaccine consisting of fentanyl hapten conjugated to tetanus toxoid or keyhole limpet hemocyanin carrier protein, and immunization with these vaccines reduced fentanyl biodistribution to the brain, and blunted its antinociceptive effects and respiratory depression in rodents ${ }^{140,141}$. Moreover, the conjugate vaccine stimulated the endogenous generation of antibodies with high affinity for a variety of fentanyl analogs ${ }^{140}$, and was shown to blunt fentanyl reinforcement ${ }^{142}$. A recent study screened and purified monoclonal antibodies (mAbs) from vaccinated mice, and found that the $6 \mathrm{~A} 4 \mathrm{mAb}$ prevented the acute lethality of fentanyl, and reversed both fentanyl and carfentanilinduced antinociception as effective as naloxone ${ }^{143}$. These findings suggest that immunopharmacotherapies including active vaccine or its combination with passive $\mathrm{mAb}$ may be potential and promising treatment strategies to address the current opioid crisis. Accumulating evidence also implicate the dysbiosis of gut microbiome in the pathophysiology of drug addiction, however data regarding fentanyl use is rare ${ }^{144}$. Manipulating the compositions of the gut microbiome or its products may guide new adjuvant therapies for opioid addiction in the future.

A United States FDA Risk Evaluation and Mitigation Strategy (REMS) program was also implemented to assess transmucosal immediate-release fentanyls (TIRFs) and found that substantial rates of TIRFs were prescribed inappropriately ${ }^{145,146}$. With the findings of deficiencies in the structure and administration of TIRFs, the development of other REMSs is needed to ensure the safe and appropriate use of approved drugs, especially dangerous opioid drugs ${ }^{147}$.

In conclusion, the crisis of opioid-related overdoses, especially fentanyl and its analogs, is a major threat to both individual and public health. Respiratory depression, cardiovascular effects, and neuropsychiatric symptoms are 
associated with fentanyl overdose and lethality. Naloxone is the standard rescue drug for fentanyl overdose, but its efficacy is inconsistent. Further clinical research is needed to optimize individualized medication-assisted treatments in patients who overdose on fentanyl and its analogs. To address the social, economic, and health problems that are associated with fentanyl and its analogs, coordinated efforts are needed to implement large-scale harm reduction strategies (e.g., naloxone distribution, innovative studies, and the development of novel drugs).

\section{Acknowledgements}

This work was supported in part by the National Natural Science Foundation of China (nos. 81701312 and 81521063).

\begin{abstract}
Author details
${ }^{1}$ National Institute on Drug Dependence and Beijing Key Laboratory of Drug Dependence, Peking University, 100191 Beijing, China. ${ }^{2}$ Peking University Sixth Hospital, Peking University Institute of Mental Health, NHC Key Laboratory of Mental Health (Peking University), National Clinical Research Center for Mental Disorders (Peking University Sixth Hospital), 100191 Beijing, China. ${ }^{3}$ PekingTsinghua Center for Life Sciences and PKU-IDG/McGovern Institute for Brain Research, Peking University, 100871 Beijing, China
\end{abstract}

\section{Conflict of interest}

The authors declare that they have no conflict of interest.

\section{Publisher's note}

Springer Nature remains neutral with regard to jurisdictional claims in published maps and institutional affiliations.

Received: 25 June 2019 Revised: 10 October 2019 Accepted: 20 October 2019

Published online: 11 November 2019

\section{References}

1. Poklis, A. Fentanyl: a review for clinical and analytical toxicologists. J. Toxicol. Clin. Toxicol. 33, 439-447 (1995).

2. Clotz, M. A. \& Nahata, M. C. Clinical uses of fentanyl, sufentanil, and alfentanil. Clin. Pharm. 10, 581-593 (1991).

3. Aldington, D. \& Jagdish, S. The fentanyl 'lozenge' story: from books to battlefield. J. R. Army Med. Corps 160, 102-104 (2014).

4. Stanley, T. H. The fentanyl story. J. Pain 15, 1215-1226 (2014).

5. Schug, S. A. \& Ting, S. Fentanyl formulations in the management of pain: an update. Drugs 77, 747-763 (2017).

6. Henderson, G. L. Fentanyl-related deaths: demographics, circumstances, and toxicology of 112 cases. J. Forensic Sci. 36, 422-433 (1991).

7. Hibbs, J., Perper, J. \& Winek, C. L. An outbreak of designer drug-related deaths in Pennsylvania. JAMA 265, 1011-1013 (1991).

8. Jalal, $\mathrm{H}$. et al. Changing dynamics of the drug overdose epidemic in the United States from 1979 through 2016. Science 361, eaau1184 (2018).

9. Suzuki, J. \& El-Haddad, S. A review: fentanyl and non-pharmaceutical fentanyls. Drug Alcohol Depend. 171, 107-116 (2017).

10. Colon-Berezin, C., Nolan, M. L., Blachman-Forshay, J. \& Paone, D. Overdose deaths involving fentanyl and fentanyl analogs-New York City, 2000-2017. Morb. Mortal. Wkly Rep. 68, 37-40 (2019).

11. Nolan, M. L., Shamasunder, S., Colon-Berezin, C., Kunins, H. V. \& Paone, D. Increased presence of fentanyl in cocaine-involved fatal overdoses: implications for prevention. J. Urban Health 96, 49-54 (2019).

12. LaRue, L. et al. Rate of fentanyl positivity among urine drug test results positive for cocaine or methamphetamine. JAMA Netw. Open 2, e192851 (2019).

13. Hedegaard, H., Warner, M. \& Minino, A. M. Drug overdose deaths in the United States, 1999-2016. NCHS Data Brief 294, 1-8 (2017).
14. Pardo, B. \& Reuter, P. Facing fentanyl: should the USA consider trialling prescription heroin? Lancet Psychiatry 5, 613-615 (2018).

15. Lyden, J. \& Binswanger, I. A. The United States opioid epidemic. Semin. Perinatol. 43, 123-131 (2019).

16. JonesC. M., EinsteinE. B. \& ComptonW. M. Changes in synthetic opioid involvement in drug overdose deaths in the United States, 2010-2016. JAMA 319, 1819-1821 (2018)

17. Scholl, L., Seth, P., Kariisa, M., Wilson, N. \& Baldwin, G. Drug and opioidinvolved overdose deaths-United States, 2013-2017. Morb. Mortal. Wkly Rep. 67, 1419-1427 (2018).

18. Ahn, J. S. et al. Transdermal buprenorphine and fentanyl patches in cancer pain: a network systematic review. J. Pain Res. 10, 1963-1972 (2017).

19. Mystakidou, K., Katsouda, E., Parpa, E., Vlahos, L. \& Tsiatas, M. L. Oral transmucosal fentanyl citrate: overview of pharmacological and clinical characteristics. Drug Deliv. 13, 269-276 (2006).

20. Katz, P., Takyar, S., Palmer, P. \& Liedgens, H. Sublingual, transdermal and intravenous patient-controlled analgesia for acute post-operative pain: systematic literature review and mixed treatment comparison. Curr. Med. Res. Opin. 33, 899-910 (2017).

21. Frank, R. G. \& Pollack, H. A. Addressing the fentanyl threat to public health. N. Engl. J. Med. 376, 605-607 (2017).

22. Smialek, J. E., Levine, B., Chin, L., Wu, S. C. \& Jenkins, A. J. A fentanyl epidemic in Maryland 1992. J. Forensic Sci. 39, 159-164 (1994).

23. Algren, D. A. et al. Fentanyl-associated fatalities among illicit drug users in Wayne County, Michigan (July 2005-May 2006). J. Med. Toxicol. 9, 106-115 (2013).

24. Ostling, P. S. et al. America's opioid epidemic: a comprehensive review and look into the rising crisis. Curr. Pain Headache Rep. 22, 32 (2018).

25. Fischer, B., Vojtila, L. \& Rehm, J. The 'fentanyl epidemic' in Canada-some cautionary observations focusing on opioid-related mortality. Prev. Med. 107, 109-113 (2018).

26. Phalen, P., Ray, B., Watson, D. P., Huynh, P. \& Greene, M. S. Fentanyl related overdose in Indianapolis: estimating trends using multilevel Bayesian models. Addict. Behav. 86, 4-10 (2018).

27. Jones, A. A. et al. Rapid change in fentanyl prevalence in a community-based, high-risk sample. JAMA Psychiatry 75, 298-300 (2018).

28. Roxburgh, A. et al. Trends in heroin and pharmaceutical opioid overdose deaths in Australia. Drug Alcohol Depend. 179, 291-298 (2017).

29. Mckeown, H. E., Rook, T. J., Pearson, J. R. \& Jones, O. A. H. Is Australia ready for fentanyl? Sci. Justice 58, 366-371 (2018).

30. O'Donnell, J. K., Halpin, J., Mattson, C. L., Goldberger, B. A. \& Gladden, R. M. Deaths involving fentanyl, fentanyl analogs, and U-4770010 states, July-December 2016. Morb. Mortal. Wkly Rep. 66, 1197-1202 (2017).

31. Gomes, T. et al. Contributions of prescribed and non-prescribed opioids to opioid related deaths: population based cohort study in Ontario, Canada. BMJ 362, k3207 (2018).

32. Daniulaityte, R. et al. Trends in fentanyl and fentanyl analogue-related overdose deaths - Montgomery County, Ohio, 2015-2017. Drug Alcohol Depend. 198, 116-120 (2019).

33. Bijral, P., Hayhurst, K. P., Bird, S. M. \& Millar, T. Prevalence of recent fentanyl use among treated users of illicit opioids in England: based on piloted urine drug screens. Clin. Toxicol. 57, 368-371 (2019).

34. Hikin, L., Smith, P. R., Ringland, E., Hudson, S. \& Morley, S. R. Multiple fatalities in the North of England associated with synthetic fentanyl analogue exposure: detection and quantitation a case series from early 2017. Forensic Sci. Int. 282, 179-183 (2018)

35. Blachman-Forshay, J., Nolan, M. L., McAteer, J. M. \& Paone, D. Estimating the risk of exposure to fentanyl in New York city: testing drug residue in used syringes. Am. J. Public Health 108, 1666-1668 (2018).

36. Hayashi, K. et al. Substance use patterns associated with recent exposure to fentanyl among people who inject drugs in Vancouver, Canada: a crosssectional urine toxicology screening study. Drug Alcohol Depend. 183, 1-6 (2018).

37. Park, J. N., Weir, B. W., Allen, S. T., Chaulk, P. \& Sherman, S. G. Fentanylcontaminated drugs and non-fatal overdose among people who inject drugs in Baltimore. Md. Harm Reduct. J. 15, 34 (2018).

38. Uuskula, A. et al. Non-fatal overdoses and related risk factors among people who inject drugs in St. Petersburg, Russia and Kohtla-Jarve, Estonia. BMC Public Health 15, 1255 (2015). 
39. Geddes, L., Iversen, J., Memedovic, S. \& Maher, L. Intravenous fentanyl use among people who inject drugs in Australia. Drug Alcohol Rev. 37(Suppl. 1), S314-S322 (2018).

40. Latimer, J., Ling, S., Flaherty, I., Jauncey, M. \& Salmon, A. M. Risk of fentanyl overdose among clients of the Sydney Medically Supervised Injecting Centre. Int J. Drug Policy 37, 111-114 (2016).

41. Dowell, D., Noonan, R. K. \& Houry, D. Underlying factors in drug overdose deaths. JAMA 318, 2295-2296 (2017).

42. Giorgetti, A., Centola, C. \& Giorgetti, R. Fentanyl novel derivative-related deaths. Hum. Psychopharmacol. 32, e2605 (2017).

43. Amlani, A. et al. Why the FUSS (Fentanyl Urine Screen Study)? A crosssectional survey to characterize an emerging threat to people who use drugs in British Columbia, Canada. Harm Reduct. J. 12, 54 (2015).

44. Arfken, C. L., Suchanek, J. \& Greenwald, M. K. Characterizing fentanyl use in methadone-maintained clients. J. Subst. Abus. Treat. 75, 17-21 (2017).

45. Cicero, T. J., Ellis, M. S. \& Kasper, Z. A. Increases in self-reported fentanyl use among a population entering drug treatment: the need for systematic surveillance of illicitly manufactured opioids. Drug Alcohol Depend. 177 101-103 (2017).

46. Strike, C. \& Watson, T. M. Losing the uphill battle? Emergent harm reduction interventions and barriers during the opioid overdose crisis in Canada. Int. J. Drug Policy. https://doi.org/10.1016/j.drugpo.2019.02.005 (2019).

47. Krieger, M. S. et al. High willingness to use rapid fentanyl test strips among young adults who use drugs. Harm Reduct. J. 15, 7 (2018).

48. Kennedy, M. C. et al. Willingness to use drug checking within future supervised injection services among people who inject drugs in a mid-sized Canadian city. Drug Alcohol Depend. 185, 248-252 (2018).

49. Bergh, M. S., Bogen, I. L., Wilson, S. R. \& Oiestad, A. M. L. Addressing the fentanyl analogue epidemic by multiplex UHPLC-MS/MS analysis of whole blood. Ther. Drug Monit. 40, 738-748 (2018).

50. Palamar, J. J. et al. Testing hair for fentanyl exposure: a method to inform harm reduction behavior among individuals who use heroin. Am. J. Drug Alcohol Abus. 45, 90-96 (2019).

51. Fogarty, M. F., Papsun, D. M. \& Logan, B. K. Analysis of fentanyl and 18 novel fentanyl analogs and metabolites by LC-MS-MS, and report of fatalities associated with methoxyacetylfentanyl and cyclopropylfentanyl. J. Anal. Toxicol. 42, 592-604 (2018).

52. Griswold, M. K. et al. A novel oral fluid assay (LC-QTOF-MS) for the detection of fentanyl and clandestine opioids in oral fluid after reported heroin overdose. J. Med. Toxicol. 13, 287-292 (2017)

53. Angelini, D. J. et al. Evaluation of a lateral flow immunoassay for the detection of the synthetic opioid fentanyl. Forensic Sci. Int. 300, 75-81 (2019).

54. Frisoni, P. et al. Novel synthetic opioids: the pathologist's point of view. Brain Sci. 8, E170 (2018).

55. Gaskey, N. J., Ferriero, L., Pournaras, L. \& Seecof, J. Use of fentanyl markedly increases nausea and vomiting in gynecological short stay patients. AANA J. 54, 309-311 (1986)

56. Yang, Q. et al. Efficacy and adverse effects of transdermal fentanyl and sustained-release oral morphine in treating moderate-severe cancer pain in Chinese population: a systematic review and meta-analysis. J. Exp. Clin. Cancer Res. 29, 67 (2010).

57. Wei, G., Moss, J. \& Yuan, C. S. Opioid-induced immunosuppression: is it centrally mediated or peripherally mediated? Biochem. Pharm. 65, 1761-1766 (2003).

58. Sacerdote, P. Opioid-induced immunosuppression. Curr. Opin. Support. Palliat. Care 2, 14-18 (2008).

59. Pergolizzi, J. et al. Opioids and the management of chronic severe pain in the elderly: consensus statement of an International Expert Panel with focus on the six clinically most often used World Health Organization Step III opioids (buprenorphine, fentanyl, hydromorphone, methadone, morphine, oxycodone). Pain. Pr. 8, 287-313 (2008).

60. Wolff, R. F. et al. Systematic review of adverse events of buprenorphine patch versus fentanyl patch in patients with chronic moderate-to-severe pain. Pain Manag. 2, 351-362 (2012).

61. Gil-Gouveia, R., Wilkinson, P. A. \& Kaube, H. Severe hemiplegic migraine attack precipitated by fentanyl sedation for esophagogastroscopy. Neurology $\mathbf{6 3}$, 2446-2447 (2004).

62. Hass, B. et al. Cost-effectiveness of strong opioids focussing on the long-term effects of opioid-related fractures: a model approach. Eur. J. Health Econ. 10 309-321 (2009).
63. Bruera, E. \& Pereira, J. Acute neuropsychiatric findings in a patient receiving fentanyl for cancer pain. Pain 69, 199-201 (1997).

64. Comer, S. D. \& Cahill, C. M. Fentanyl: receptor pharmacology, abuse potential, and implications for treatment. Neurosci. Biobehav. Rev. 106, 49-57 (2019).

65. Volkow, N. D., Jones, E. B., Einstein, E. B. \& Wargo, E. M. Prevention and treatment of opioid misuse and addiction: a review. JAMA Psychiatry 76, 208-216 (2019).

66. Schifano, F., Chiappini, S., Corkery, J. M. \& Guirguis, A. Assessing the 2004-2018 fentanyl misusing issues reported to an international range of adverse reporting systems. Front. Pharm. 10, 46 (2019).

67. Nadpara, P. A. et al. Risk factors for serious prescription opioid-induced respiratory depression or overdose: comparison of commercially insured and veterans health affairs populations. Pain Med. 19, 79-96 (2018).

68. Baumann, M. H., Kopajtic, T. A. \& Madras, B. K. Pharmacological research as a key component in mitigating the opioid overdose crisis. Trends Pharm. Sci. 39, 995-998 (2018).

69. Chen, J. C., Smith, E. R., Cahill, M., Cohen, R. \& Fishman, J. B. The opioid receptor binding of dezocine, morphine, fentanyl, butorphanol and nalbuphine. Life Sci. 52, 389-396 (1993).

70. Andersen, H. B., Christensen, B., Findlay, J. W. \& Jansen, J. A. Pharmacokinetics of intravenous, intrathecal and epidural morphine and fentanyl in the goat Acta Anaesthesiol. Scand. 30, 393-399 (1986).

71. Jang, Y. \& Yoburn, B. C. Evaluation of receptor mechanism mediating fentanyl analgesia and toxicity. Eur. J. Pharm. 197, 135-141 (1991).

72. Tschirhart, J. N., Li, W., Guo, J. \& Zhang, S. Blockade of the human ether a-gogo-related gene (hERG) potassium channel by fentanyl. Mol. Pharm. 95, 386-397 (2019).

73. Kiyatkin, E. A. Respiratory depression and brain hypoxia induced by opioid drugs: morphine, oxycodone, heroin, and fentanyl. Neuropharmacology 151, 219-226 (2019).

74. Dahan, A. et al. Comparison of the respiratory effects of intravenous buprenorphine and fentanyl in humans and rats. Br. J. Anaesth. 94, 825-834 (2005).

75. Solis, E. Jr., Cameron-Burr, K. T., Shaham, Y. \& Kiyatkin, E. A. Fentanyl-induced brain hypoxia triggers brain hyperglycemia and biphasic changes in brain temperature. Neuropsychopharmacology 43, 810-819 (2018).

76. Barash, J. A. et al. Acute amnestic syndrome associated with fentanyl overdose. N. Engl. J. Med. 378, 1157-1158 (2018).

77. Kofke, W. A., Garman, R. H., Stiller, R. L., Rose, M. E. \& Garman, R. Opioid neurotoxicity: fentanyl dose-response effects in rats. Anesth. Analg. 83, 1298-1306 (1996).

78. Duru, U. B. et al. An unusual amnestic syndrome associated with combined fentanyl and cocaine use. Ann. Intern. Med. 169, 662-663 (2018).

79. Taylor, R. G., Budhram, A., Lee, D. H. \& Mirsattari, S. M. Opioid-associated amnestic syndrome observed with fentanyl patch use. CMAJ 191, E337-E339 (2019).

80. Solis, E., Jr., Cameron-Burr, K. T. \& Kiyatkin, E. A. Heroin contaminated with fentanyl dramatically enhances brain hypoxia and induces brain hypothermia. eNeuro 4, ENEURO.0323-17.2017 (2017).

81. Harper, M. H., Hickey, R. F., Cromwell, T. H. \& Linwood, S. The magnitude and duration of respiratory depression produced by fentanyl and fentanyl plus droperidol in man. J. Pharm. Exp. Ther. 199, 464-468 (1976).

82. Magosso, E., Ursino, M. \& van Oostrom, J. H. Opioid-induced respiratory depression: a mathematical model for fentanyl. IEEE Trans. Biomed. Eng. $\mathbf{5 1}$ 1115-1128 (2004).

83. Goetz, A. M. et al. Adult respiratory distress syndrome associated with epidural fentanyl infusion. Crit. Care Med. 22, 1579-1583 (1994)

84. Chen, S. W., Maguire, P. A., Davies, M. F., Beatty, M. F. \& Loew, G. H. Evidence for mu1-opioid receptor involvement in fentanyl-mediated respiratory depression. Eur. J. Pharm. 312, 241-244 (1996).

85. Ren, J., Ding, X. \& Greer, J. J. Activating alpha4beta2 nicotinic acetylcholine receptors alleviates fentanyl-induced respiratory depression in rats. Anesthesiology 130, 1017-1031 (2019).

86. Roozekrans, M. et al. Two studies on reversal of opioid-induced respiratory depression by BK-channel blocker GAL021 in human volunteers. Anesthesiology 121, 459-468 (2014).

87. Dahan, A. et al. Averting opioid-induced respiratory depression without affecting analgesia. Anesthesiology 128, 1027-1037 (2018).

88. Golder, F. J. et al. Identification and characterization of GAL-021 as a novel breathing control modulator. Anesthesiology 123, 1093-1104 (2015). 
89. Oden, R. V. \& Karagianes, T. G. Postoperative myocardial ischemia possibly masked by epidural fentanyl analgesia. Anesthesiology 74, 941-943 (1991).

90. Blair, J. R., Pruett, J. K., Crumrine, R. S. \& Balser, J. J. Prolongation of QT interval in association with the administration of large doses of opiates. Anesthesiology 67, 442-443 (1987).

91. Hilgenberg, J. C. \& Johantgen, W. C. Bradycardia after intravenous fentanyl during subarachnoid anesthesia. Anesth. Analg. 59, 162-163 (1980).

92. Zuin, M., Rigatelli, G. \& Roncon, L. Use of fentanyl during percutaneous coronary interventions: safety and drawbacks. Cardiovasc. Drugs Ther. 32, 625-632 (2018).

93. McEvoy, J. W. et al. Effect of intravenous fentanyl on ticagrelor absorption and platelet inhibition among patients undergoing percutaneous coronary intervention: the PACIFY randomized clinical trial (platelet aggregation with ticagrelor inhibition and fentanyl). Circulation 137, 307-309 (2018).

94. Ibrahim, $\mathrm{K}$. et al. Effect of intravenous fentanyl on ticagrelor absorption and platelet inhibition among patients undergoing percutaneous coronary intervention: design, rationale, and sample characteristics of the PACIFY randomized trial. Contemp. Clin. Trials 64, 8-12 (2018).

95. Takase, I., Koizumi, T., Fujimoto, I., Yanai, A. \& Fujimiya, T. An autopsy case of acetyl fentanyl intoxication caused by insufflation of 'designer drugs'. Leg. Med. 21, 38-44 (2016).

96. Poklis, J. et al. Two fatal intoxications involving butyryl fentanyl. J. Anal. Toxicol. 40, 703-708 (2016)

97. Tsuchida, H., Schubert, A., Estafanous, F. G., Brum, J. M. \& Murray, P. A. Sigma receptor activation does not mediate fentanyl-induced attenuation of muscarinic coronary contraction. Anesth. Analg. 82, 982-987 (1996).

98. Mitra, S. Opioid-induced hyperalgesia: pathophysiology and clinical implications. J. Opioid Manag. 4, 123-130 (2008).

99. Mao, J. Opioid-induced abnormal pain sensitivity: implications in clinical opioid therapy. Pain 100, 213-217 (2002).

100. Li, Z. et al. CaMKIlalpha may modulate fentanyl-induced hyperalgesia via a CeLC-PAG-RVM-spinal cord descending facilitative pain pathway in rats. PLoS ONE 12, e0177412 (2017)

101. Li, Z. et al. Activation of the extracellular signal-regulated kinase in the amygdale modulates fentanyl-induced hypersensitivity in rats. J. Pain 18, 188-199 (2017)

102. Bart, G. Maintenance medication for opiate addiction: the foundation of recovery. J. Addict. Dis. 31, 207-225 (2012).

103. Doughty, B., Morgenson, D. \& Brooks, T. Lofexidine: a newly FDA-approved, nonopioid treatment for opioid withdrawal. Ann. Pharmacother. 53, 746-753 (2019).

104. Gorodetzky, C. W. et al. A phase III, randomized, multi-center, double blind, placebo controlled study of safety and efficacy of lofexidine for relief of symptoms in individuals undergoing inpatient opioid withdrawal. Drug Alcohol Depend. 176, 79-88 (2017).

105. Kinshella, M. W., Gauthier, T. \& Lysyshyn, M. Rigidity, dyskinesia and other atypical overdose presentations observed at a supervised injection site, Vancouver, Canada. Harm. Reduct. J. 15, 64 (2018).

106. Mayer, S. et al. Characterizing fentanyl-related overdoses and implications for overdose response: findings from a rapid ethnographic study in Vancouver, Canada. Drug Alcohol Depend. 193, 69-74 (2018).

107. Floyd, C. N. \& Warren, J. B. Opioids out of control. Br. J. Clin. Pharm. 84 813-815 (2018)

108. Scheuermeyer, F. X. et al. Safety of a brief emergency department observation protocol for patients with presumed fentanyl overdose. Ann. Emerg. Med. 72, 1-8 e1 (2018).

109. Stone, A. C., Carroll, J. J., Rich, J. D. \& Green, T. C. Methadone maintenance treatment among patients exposed to illicit fentanyl in Rhode Island: safety, dose, retention, and relapse at 6 months. Drug Alcohol Depend. 192, 94-97 (2018).

110. Pendergrass, S. A., Crist, R. C., Jones, L. K., Hoch, J. R. \& Berrettini, W. H. The importance of buprenorphine research in the opioid crisis. Mol. Psychiatry 24 626-632 (2019).

111. Wakeman, S. E. et al. Impact of fentanyl use on buprenorphine treatment retention and opioid abstinence. J. Addict. Med. 13, 253-257 (2019).

112. Walker, E. A. \& Young, A. M. Differential tolerance to antinociceptive effects of mu opioids during repeated treatment with etonitazene, morphine, or buprenorphine in rats. Psychopharmacology 154, 131-142 (2001).

113. Barrett, A. C., Smith, E. S. \& Picker, M. J. Use of irreversible antagonists to determine the relative efficacy of mu-opioids in a pigeon drug discrimination procedure: comparison of beta-funaltrexamine and clocinnamox. J. Pharm Exp. Ther. 305, 1061-1070 (2003).

114. Negus, S. S., Brandt, M. R., Gatch, M. B. \& Mello, N. K. Effects of heroin and its metabolites on schedule-controlled responding and thermal nociception in rhesus monkeys: sensitivity to antagonism by quadazocine, naltrindole and beta-funaltrexamine. Drug Alcohol Depend. 70, 17-27 (2003)

115. Winger, G. \& Woods, J. H. The effects of chronic morphine on behavior reinforced by several opioids or by cocaine in rhesus monkeys. Drug Alcohol Depend. 62, 181-189 (2001).

116. Bisaga, A. What should clinicians do as fentanyl replaces heroin? Addiction. https://doi.org/10.1111/add.14522 (2019).

117. Kuczynska, K., Grzonkowski, P., Kacprzak, L. \& Zawilska, J. B. Abuse of fentanyl: an emerging problem to face. Forensic Sci. Int. 289, 207-214 (2018).

118. Fairbairn, N., Coffin, P. O. \& Walley, A. Y. Naloxone for heroin, prescription opioid, and illicitly made fentanyl overdoses: challenges and innovations responding to a dynamic epidemic. Int. J. Drug Policy 46, 172-179 (2017)

119. Rzasa Lynn, R. \& Galinkin, J. L. Naloxone dosage for opioid reversal: current evidence and clinical implications. Ther. Adv. Drug Saf. 9, 63-88 (2018).

120. Lee, J. D. et al. Comparative effectiveness of extended-release naltrexone versus buprenorphine-naloxone for opioid relapse prevention ( $\mathrm{X}: \mathrm{BOT}$ ): a multicentre, open-label, randomised controlled trial. Lancet 391, 309-318 (2018).

121. Tanum, L. et al. Effectiveness of injectable extended-release naltrexone vs daily buprenorphine-naloxone for opioid dependence: a randomized clinical noninferiority trial. JAMA Psychiatry 74, 1197-1205 (2017).

122. Bell, A., Bennett, A. S., Jones, T. S., Doe-Simkins, M. \& Williams, L. D. Amount of naloxone used to reverse opioid overdoses outside of medical practice in a city with increasing illicitly manufactured fentanyl in illicit drug supply. Subst. Abus. 40, 52-55 (2019).

123. Schumann, H., Erickson, T., Thompson, T. M., Zautcke, J. L. \& Denton, J. S. Fentanyl epidemic in Chicago, Illinois and surrounding Cook County. Clin. Toxicol. 46, 501-506 (2008).

124. Freise, K. J., Newbound, G. C., Tudan, C. \& Clark, T. P. Naloxone reversal of an overdose of a novel, long-acting transdermal fentanyl solution in laboratory Beagles. J. Vet. Pharm. Ther. 35(Suppl. 2), 45-51 (2012).

125. Greene, J. A., Deveau, B. J., Dol, J. S. \& Butler, M. B. Incidence of mortality due to rebound toxicity after 'treat and release' practices in prehospital opioid overdose care: a systematic review. Emerg. Med. J. 36, 219-224 (2019)

126. Karamouzian, M., Kuo, M., Crabtree, A. \& Buxton, J. A. Correlates of seeking emergency medical help in the event of an overdose in British Columbia, Canada: findings from the Take Home Naloxone program. Int. J. Drug Policy. https://doi.org/10.1016/j.drugpo.2019.01.006 (2019).

127. Latkin, C. A., Dayton, L., Davey-Rothwell, M. A. \& Tobin, K. E. Fentanyl and drug overdose: perceptions of fentanyl risk, overdose risk behaviors, and opportunities for intervention among people who use opioids in Baltimore, USA. Subst. Use Misuse 54, 998-1006 (2019).

128. Irvine, M. A. et al. Distribution of take-home opioid antagonist kits during a synthetic opioid epidemic in British Columbia, Canada: a modelling study. Lancet Public Health 3, e218-e225 (2018).

129. McDonald, R. Campbell, N. D. \& Strang, J. Twenty years of take-home naloxone for the prevention of overdose deaths from heroin and other opioids-conception and maturation. Drug Alcohol Depend. 178, 176-187 (2017).

130. Elzey, M. J., Fudin, J. \& Edwards, E. S. Take-home naloxone treatment for opioid emergencies: a comparison of routes of administration and associated delivery systems. Expert Opin. Drug Deliv. 14, 1045-1058 (2017).

131. Kim, H. K. Connors, N. J. \& Mazer-Amirshahi, M. E. The role of take-home naloxone in the epidemic of opioid overdose involving illicitly manufactured fentanyl and its analogs. Expert Opin. Drug Saf. 18, 465-475 (2019).

132. Yong, Z. et al. Nalmefene reverses carfentanil-induced loss of righting reflex and respiratory depression in rats. Eur. J. Pharm. 738, 153-157 (2014).

133. Palpacuer, $C$. et al. Risks and benefits of nalmefene in the treatment of adult alcohol dependence: a systematic literature review and meta-analysis of published and unpublished double-blind randomized controlled trials. PLoS Med. 12, e1001924 (2015).

134. Keating, G. M. Nalmefene: a review of its use in the treatment of alcohol dependence. CNS Drugs 27, 761-772 (2013). 
135. Castera, P. et al. Nalmefene, given as needed, in the routine treatment of patients with alcohol dependence: an interventional, open-label study in primary care. Eur. Addict. Res. 24, 293-303 (2018).

136. Fragale, J. E., Pantazis, C. B., James, M. H. \& Aston-Jones, G. The role of orexin-1 receptor signaling in demand for the opioid fentanyl. Neuropsychopharmacology 44, 1690-1697 (2019).

137. Obeng, S. et al. Characterization of 17-cyclopropylmethyl-3,14beta-dihydroxy4,5alpha-epoxy-6alpha-(indole-7-carboxami do)morphinan (NAN) as a novel opioid receptor modulator for opioid use disorder treatment. ACS Chem. Neurosci. 10, 2518-2532 (2019).

138. Nozari, A. et al. Prolonged therapy with the anticonvulsant carbamazepine leads to increased plasma clearance of fentanyl. J. Pharm. Pharm. 71, 982-987 (2019).

139. Prinsloo, G., Ahamad, K. \& Socias Me, Md M. Successful treatment with slowrelease oral morphine following afentanyl-related overdose: a case report Subst. Abus. https://doi.org/10.1080/08897077.2019.1576086 (2019).

140. Bremer, P. T. et al. Combatting synthetic designer opioids: a conjugate vaccine ablates lethal doses of fentanyl class drugs. Angew. Chem. Int. Ed. Engl. 55, 3772-3775 (2016).
141. Raleigh, M. D. et al. A fentanyl vaccine alters fentanyl distribution and protects against fentanyl-induced effects in mice and rats. J. Pharm. Exp. Ther. $\mathbf{3 6 8}$ 282-291 (2019).

142. Townsend, E. A. et al. Conjugate vaccine produces long-lasting attenuation of fentanyl vs. food choice and blocks expression of opioid withdrawalinduced increases in fentanyl choice in rats. Neuropsychopharmacology $\mathbf{4 4}$, 1681-1689 (2019).

143. Smith, L. C. et al. Monoclonal antibodies for combating synthetic opioid intoxication. J. Am. Chem. Soc. 141, 10489-10503 (2019).

144. Meckel, K. R. \& Kiraly, D. D. A potential role for the gut microbiome in substance use disorders. Psychopharmacology 236, 1513-1530 (2019).

145. Rollman, J. E. et al. Assessment of the FDA Risk Evaluation and Mitigation Strategy for transmucosal immediate-release fentanyl products. JAMA 321, 676-685 (2019).

146. Fleischman, W., Auth, D., Shah, N. D., Agrawal, S. \& Ross, J. S. Association of a Risk Evaluation and Mitigation Strategy program with transmucosal fentanyl prescribing. JAMA Netw. Open 2, e191340 (2019).

147. Sarpatwari, A. \& Curfman, G. Mitigating health risks of prescription drugs: lessons from FDA oversight of opioid products. JAMA 321, 651-653 (2019). 